\title{
Efecto del ajuste estructural sobre la situación de salud en América Latina y el Caribe, 1980-2000
}

\author{
Álvaro Franco-Giraldo, ${ }^{1}$ Marco Palma ${ }^{2}$ y Carlos Álvarez-Dardet ${ }^{3}$
}

Forma de citar Franco-Giraldo A, Palma M, Álvarez-Dardet C. Efecto del ajuste estructural sobre la situación de salud en América Latina y el Caribe, 1980-2000. Rev Panam Salud Publica. 2006;19(5):291-9.

RESUMEN Objetivo. Evaluar el impacto del ajuste estructural sobre los indicadores de salud en América Latina y el Caribe durante el período de 1980-2000.

Métodos. Se trata de un estudio ecológico. Los datos del gasto público y del producto interno bruto per cápita (PIBpc) se tomaron del Banco Mundial y los de la esperanza de vida (EV) y mortalidad infantil (MI), de la Organización Mundial de la Salud. El ajuste estructural (adelgazamiento del Estado) se evaluó mediante el cambio en el consumo de gobierno (la reducción del gasto público) en los países de América Latina y el Caribe en la década de 19801990. El cambio en los indicadores de salud se midió por la variación porcentual de la EV y la MI. Se compararon estas variaciones en América Latina y el Caribe con las observadas en diferentes grupos de países de otras regiones del mundo durante el período de 1980-2000. Se determinaron las asociaciones entre la reducción del gasto público y los indicadores de salud mediante la prueba de Pearson. Para estimar el efecto de los cambios sobre la salud se construyó un modelo de regresión lineal multifactorial ajustado según el PIBpc.

Resultados. Se observó una ralentización del incremento de la EV y del descenso de la MI en América Latina y el Caribe, especialmente en la década de 1980-1990. Se encontraron asociaciones significativas entre la variación de los indicadores de salud y el cambio del gasto público en todos los grupos de países analizados. Al ajustar el modelo de regresión múltiple, las asociaciones solo se mantuvieron en América Latina y el Caribe.

Conclusiones. En la década de 1980, el ajuste de las políticas macroeconómicas tuvo un efecto negativo sobre los indicadores sociales, específicamente sobre los relacionados con la situación de salud en América Latina y el Caribe. Estos efectos se siguieron observando durante la década siguiente.

Palabras clave Reforma del Estado, reducción del gasto público, impacto en el estado de salud, América Latina, región del Caribe.

\footnotetext{
Facultad Nacional de Salud Pública, Universidad de Antioquia, Medellín, Colombia. La correspondencia se debe dirigir a Álvaro Franco-Giraldo, Facultad Nacional de Salud Pública, Universidad de Antioquia, calle 62 No. 52-59, oficina 305, Medellín, Colombia. Correos electrónicos: alvarofranco giraldo@hotmail.com y afranco@guajiros.udea. edu.co

2 Universidad Autónoma de Yucatán, Mérida, México.

3 Universidad de Alicante, Alicante, España.
}

Los programas de ajuste estructural comenzaron en América Latina en la década de 1980 y se extendieron hasta el final del siglo pasado. Los países latinoamericanos han sido los que en mayor grado se han visto forzados a aceptar los postulados del Estado mí- nimo $(1,2)$, preconizados por los defensores del neoliberalismo (3). En América Latina predomina el proceso de desmonte de las entidades y servicios públicos y la flexibilización laboral (4), llegando al extremo de violar leyes basadas en principios sociales univer- 
sales, según denuncian los opositores a la globalización económica (5).

Las reformas estructurales de tipo económico han tenido un fuerte impacto en América Latina a partir de la década de 1980, según se desprende de la evolución de los indicadores económicos y sociales. Estas políticas se derivaron de acuerdos con organismos financieros internacionales y están asociadas con dictámenes que posteriormente se denominarían "Consenso de Washington" (6). En el presente estudio se denomina "ajuste estructural" a todo el conjunto de reformas de corte neoliberal.

La crisis del Estado se venía gestando desde tiempo atrás y se atribuía a las llamadas ineficiencias estatales y al excesivo gasto público sin destino específico, agravado por el alto índice de inflación. Además, el gasto social no lograba solucionar los graves problemas de la población y la inaccesibilidad de los servicios públicos de salud, que fue notable durante la última fase del llamado Estado de Bienestar, especialmente en los Estados "desarrollistas" de América Latina. Aun sin las reformas, se hacía necesario corregir muchas de las falencias que arrastraba el sector público, corregir la destinación del gasto social y hacer un uso más eficiente de los recursos. En el siglo XX, la expansión del Estado fue enorme en proyectos de bienestar, programas sociales y estrategias para el desarrollo, pero no tuvo una orientación adecuada. Eran, finalmente, las expresiones de una crisis derivada del agotamiento del modelo anterior, definida por algunos como "exceso de democracia, retardación de la economía y déficit fiscal" (7).

Pero los programas de ajuste estructural y la crisis económica agravaron la pobreza durante las dos últimas décadas debido a los recortes en empleos y en el gasto social (8), por lo que resultó peor la medicina que la enfermedad. No obstante, el Banco Mundial (9) aseguraba que la intervención estatal había fracasado y que las economías se debían inclinar en favor de los mecanismos de mercado. Por su parte, Mesa-Lagos (8) analizó y caracterizó los aspectos económicos de esta etapa como: el predomi- nio de la teoría neoliberal, la expansión del mercado, la reducción del tamaño y de las funciones del Estado, la desregulación, el recorte del gasto y de empleos públicos, la privatización de empresas y de servicios públicos, el estímulo a la competencia y la apertura al comercio mundial y al capital extranjero, además de otros fenómenos asociados con la globalización económica. En el aspecto social, se agravó la crisis de los seguros sociales y se restringió aun más la antigua asistencia social.

En América Latina cayó el producto interno bruto per cápita (PIBpc) promedio, especialmente en Argentina, Bolivia y Perú, y aumentó la inflación, aunque con una posterior recuperación en la década de 1990. En lo social se destaca la caída del salario mínimo, especialmente en Argentina, México y Perú, y el aumento de las tasas de desempleo en países como Chile, Colombia y Uruguay; la pobreza se incrementó, especialmente en Bolivia y Perú; la distribución del ingreso se hizo más desigual en casi todos los países, particularmente en Chile y Bolivia durante la década de 1980 y posteriormente en Colombia y Brasil. Estos dos países, junto con Chile y Paraguay, configuran el espectro de mayor inequidad interna en América Latina, además de recibir el mayor impacto social negativo en esa década; en Colombia, ese efecto se sintió en la década siguiente.

Si bien esa experiencia de ajustes estructurales ha sido estudiada en relación con sus implicaciones sociales y económicas $(6,8,10)$, no se ha hecho un análisis integral de su impacto en el sector de la salud (11). Existen solo algunos estudios parciales al respecto, que señalan que una de las consecuencias de las reformas económicas ha sido el desmonte de los sistemas de seguridad social y de los programas basados en la solidaridad sistémica y en la protección social, como la salud, la educación y el trabajo (12). Kickbusch y colaboradores (13) publicaron un análisis teórico en el que mencionan otros factores propiciados por la globalización que influyen sobre la salud de las poblaciones, tales como el aumento de la interdependencia entre los países, la mayor influencia de los factores tem- porales y espaciales y la presencia de nuevos actores y alianzas. La situación de la salud pública se agravó porque los Estados perdieron el poder de actuar en relación con algunos factores determinantes fundamentales de la salud, como el comportamiento de los mercados internacionales, los nuevos estilos de vida y de consumo, el comercio de bienes y servicios de salud o la contaminación ambiental generalizada. De acuerdo con nuestra hipótesis de trabajo, estos factores agravaron la crisis latinoamericana en los últimos 20 años del siglo pasado.

Análisis más generales concluyeron, mediante el estudio de las relaciones de la salud con los factores determinantes macrosociales según diferentes interpretaciones, que las desigualdades y los problemas sanitarios aumentaron a finales del siglo pasado (14-22). En los últimos años se ha vuelto práctica común el análisis económico sanitario basado en la medición del efecto del gasto público y privado en la salud (23, 24). Sin embargo, algunos investigadores han encontrado que el impacto del gasto público en el sector de la salud es muy pequeño en comparación con el de los factores socioeconómicos, como el ingreso per cápita, la inequidad en la distribución del ingreso y el nivel de educación, entre otros (25).

El ajuste estructural ha implicado la reducción del gasto público global de los Estados $(26,27)$, especialmente en América Latina y el Caribe, lo que ha permitido comparar los indicadores de salud y explorar las implicaciones negativas de la reducción del tamaño del Estado sobre la situación de la salud en los países del mundo, en general (28). Aunque en el decenio de 1990 empezó la recuperación económica, ella no se tradujo suficientemente en mejoras sociales. Más adelante se presentaron otras crisis, como la mexicana y la argentina.

El presente estudio se diseñó con el propósito de evaluar el impacto del ajuste estructural sobre los indicadores de salud en América Latina y el Caribe durante el período de 1980-2000. A pesar de que el estudio se centró fundamentalmente en la década de 1980, también se exploraron algunas asociaciones 
entre diversos factores socioeconómicos e indicadores de salud y su comportamiento en la década siguiente.

\section{MATERIALES Y MÉTODOS}

Se realizó un estudio ecológico y se compararon las variaciones de los indicadores estudiados en diferentes regiones del mundo, según la clasificación utilizada en el Informe Anual del Fondo Monetario Internacional (FMI) (29). Finalmente se concentró el análisis en los países de América Latina, tanto individualmente como en su conjunto.

\section{Población y fuentes}

El estudio abarcó 33 países de América Latina y el Caribe: Antigua y Barbuda, Argentina, Bahamas, Barbados, Belice, Bolivia, Brasil, Chile, Colombia, Costa Rica, Cuba, Dominica, Ecuador, El Salvador, Granada, Guatemala, Guyana, Haití, Honduras, Jamaica, México, Nicaragua, Panamá, Paraguay, Perú, República Dominicana, Saint Kitts y Nevis, San Vicente y las Granadinas, Santa Lucía, Suriname, Trinidad y Tabago, Uruguay y Venezuela. Para los efectos comparativos, el análisis se extendió a otros países y regiones del mundo hasta alcanzar la cifra de 177 países en la muestra. Los datos económicos y financieros se tomaron del Banco Mundial (27) y del FMI (29). El gasto público se expresó como el gasto gubernamental total y el consumo del gobierno, mientras que el producto interno bruto se expresó per cápita (PIBpc). Los indicadores de salud empleados fueron la esperanza de vida al nacer (EV) y la mortalidad infantil (MI) y los datos nacionales se obtuvieron de la Organización Mundial de la Salud (30), las Naciones Unidas (31) y el Programa de las Naciones Unidas para el Desarrollo (PNUD) (32).

\section{Variables e indicadores}

El tamaño del Estado se midió mediante el porcentaje del gasto público nacional con respecto al PIBpc en el período 1980-2000. En general, el tamaño del sector público se mide por la relación entre el gasto público (gasto público $=$ consumo + inversión) y el gasto total o el producto total de la economía. El consumo público se considera equivalente al costo salarial de la administración, ya que aunque es más restrictivo, es más exacto que el valor de los beneficios percibidos por el gasto público. El gasto gubernamental total equivale a la suma del consumo, el monto de las inversiones, los intereses, las transferencias y las subvenciones. Las pensiones y prestaciones pueden formar parte del gasto gubernamental. El gasto público total está constituido por el gasto gubernamental, los gastos locales y los extra presupuestarios. Algunas formas de privatización subvencionadas por el Estado se consideran como una ampliación del tamaño del Estado por implicar un gasto público, como sucedió en algunos países de América Latina ${ }^{4}$.

Para determinar el ajuste estructural se utilizó la variable cambio en el consumo de gobierno, como el adelgazamiento del Estado, medido por la diferencia del gasto público (consumo de gobierno) como porcentaje del PIB entre un año y otro, por país, en el período 1980-2000. Esta fue la variable más importante en la investigación, ya que permitió establecer la relación de los cambios socioeconómicos con la salud.

El cambio en los indicadores de salud se estableció mediante la variación porcentual de la MI y de la EV en cada quinquenio.

\section{Métodos de análisis}

Los datos se agruparon por quinquenio (los años terminados en 8, 9, 1 y 2 se agruparon junto con los años terminados en 0 ; los años terminados en $3,4,6$ y 7 se agrupan junto con los años terminados en 5 ). De esta forma se ob-

\footnotetext{
4 Para obtener mayor información acerca de la metodología utilizada para construir este indicador del tamaño del Estado se puede consultar un documento publicado por el Consejo Económico y Social de las Naciones Unidad (26).
}

tuvieron series de datos para 1980, $1985,1990,1995$ y 2000. Con los datos de 2000 se realizó un análisis exploratorio mediante cruces simples de cada uno de los indicadores entre sí y se compararon las asociaciones mediante la prueba de Pearson y las medidas de tendencia central. En esta etapa se siguió un modelo de análisis bifactorial de las variables económicas y políticas (variables explicativas o independientes) y de las variables de salud (variables dependientes).

Posteriormente, con los datos de 1980 a 2000 se realizó un análisis de la variación porcentual por quinquenio de cada uno de los indicadores de salud en las dos décadas estudiadas. Se obtuvieron estadísticos descriptivos de todos los indicadores para cada período y se realizaron comparaciones entre los diferentes períodos (1980-1985, 19851990, 1990-1995 y 1995-2000) mediante la variación porcentual quinquenal (es decir, el promedio del porcentaje de variación del indicador para cada período analizado). Se aplicaron la prueba de la $t$ de Student para comparar las medias de los indicadores de salud en diferentes períodos y la prueba no paramétrica de Friedman para comparar las variaciones porcentuales de los indicadores de salud.

Se compararon los indicadores sanitarios y socioeconómicos de América Latina con los de otros grupos de países durante el mismo período. También se evaluó, mediante la prueba de Pearson, la correlación entre la reducción del gasto público y los cambios en los indicadores de salud en los diferentes grupos de países. Finalmente se construyó un modelo de regresión lineal multifactorial para calcular el efecto del adelgazamiento del Estado sobre los indicadores de salud (EV y MI), ajustados por el PIBpc, en América Latina durante la década de 1980-1990.

\section{RESULTADOS}

En el período estudiado, América Latina tuvo uno de los gastos públicos más bajos del mundo, alrededor de $20 \%$ de su PIB. A pesar de ello, fue una 
de las regiones donde más rápido se evidenció el ajuste, ya que se redujo aun más el gasto público durante la década de 1980 antes de entrar en cierta estabilidad (figura 1).

A pesar del descenso del PIBpc durante la década de 1980, es notoria la disminución del tamaño del Estado en América Latina. En los países asiáticos en desarrollo (como China, Singapur y Viet Nam) y en los países en transición económica (como Bosnia y Herzegovina, Kirguistán, Lituania, Polonia, Rumania y Rusia), ese proceso comenzó una década después. En los demás grupos de países, a pesar del discurso proclive al achicamiento del Estado, se experimentaron pocos cambios de tendencia en relación con el gasto y consumo gubernamentales.

\section{Comportamiento de los indicadores de salud}

En general se observó la ralentización de la tendencia al aumento de la EV y al descenso de la MI en el mundo. Estos cambios son estadísticamente significativos según pruebas de contraste no paramétricas (como la prueba de Friedman), tanto para el porcentaje de la variación quinquenal de la EV $\left(\chi^{2}=62,529 ; P<0,001\right)$ como de la MI $\left(\chi^{2}=27.250 ; P<0,001\right)$ en todos los países estudiados (33).

Esa ralentización de la variación se hace más visible si se analizan los cambios por grupos de países (figura 2). En América Latina, las variaciones son mayores en el período de 1980-1990 que en el siguiente, aunque persiste el retraso. Otros grupos de países expresaron con mayor intensidad el efecto de la crisis durante la década siguiente.

En el período estudiado, la EV sufrió menos variaciones en América Latina que en la mayoría de los otros grupos de países en desarrollo, entre ellos los africanos, y se observó un menor crecimiento, es decir, una mayor ralentización en el incremento de este indicador.

La MI refleja de manera más clara y contundente el deterioro de las condiciones de salud en los países latinoamericanos que en otros países, ya que el

FIGURA 1. Tamaño del Estado según el porcentaje del producto interno bruto dedicado al gasto público (GP \% PIB) en diferentes grupos de países, 1980-2000

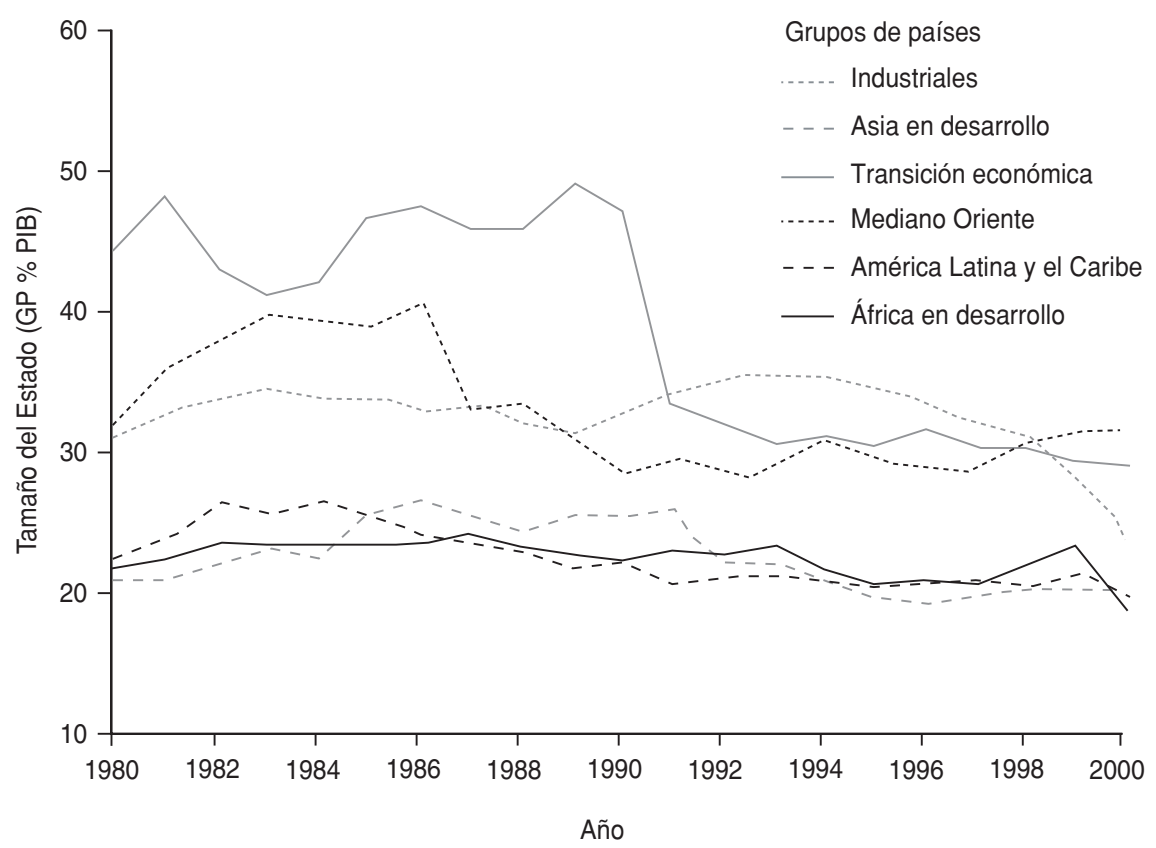

Fuente: Datos del Fondo Monetario Internacional (29).

descenso de las tasas de MI en este grupo de países fue menor durante la década de 1980 que en todas las demás. En la década siguiente, este fenómeno se agravó en la mayoría de las regiones del mundo, y no solo se observó la ralentización de la tendencia a mejorar de las tasas de EV y MI, sino la inversión de esa tendencia en algunos países.

\section{Algunas asociaciones}

Los indicadores de salud presentan una asociación con el tamaño del Estado, específicamente en los países de América Latina (figura 3). La EV aumenta a medida que aumenta el tamaño del Estado, mientras que la MI disminuye.

En general, según muestra la correlación de Pearson, existe una asociación significativa entre los indicadores de salud y el adelgazamiento del Estado en todo el mundo (cuadro 1).

Si bien estas asociaciones también se dan en otros países y en algunos casos resultan muy similares a las encontra- das en América Latina, cuando se aplica el modelo de regresión lineal multifactorial y se ajusta según el $\mathrm{PIBpc}$, las asociaciones encontradas en los otros grupos de países pierden su significación estadística y esta solo se mantiene en América Latina durante el período de 1980-1990, incluso con mayor fuerza estadística (cuadro 2).

Por ejemplo, aunque la asociación simple de la MI con el cambio en el consumo del gobierno puede ser mayor de $50 \%$, se debe tener en cuenta su colinealidad con el PIBpc. De ahí la importancia de hacer el ajuste según el PIBpc y de aplicar el modelo de regresión lineal multifactorial, tanto para la MI como para la EV. De esta forma se obtienen las siguientes ecuaciones:

$$
\begin{gathered}
\mathrm{MI}=42,82-0,002 \cdot \mathrm{PIBpc}-0,554 \\
\quad(\text { cambio en el gasto público) } \\
\mathrm{EV}=65,74+0,001 \cdot \mathrm{PIBpc}+0,072 \\
(\text { cambio en el gasto público) }
\end{gathered}
$$

En ambos casos, el coeficiente de regresión ajustado según los otros factores es estadísticamente significativo $(P<0,01)$. 
FIGURA 2. Variación porcentual quinquenal promedio de dos indicadores de salud, por grupos de países, $1980-2000$

A. Esperanza de vida (EV), en años

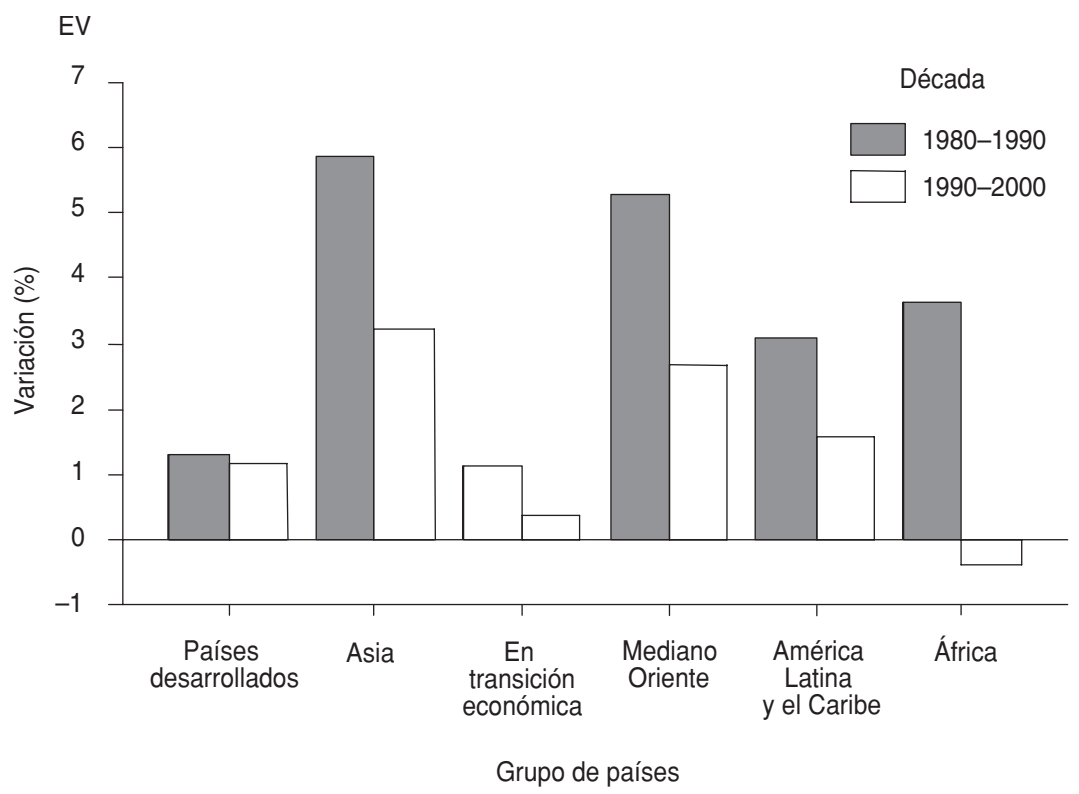

B. Tasa de mortalidad infantil (TMI) por 1000 nacidos vivos

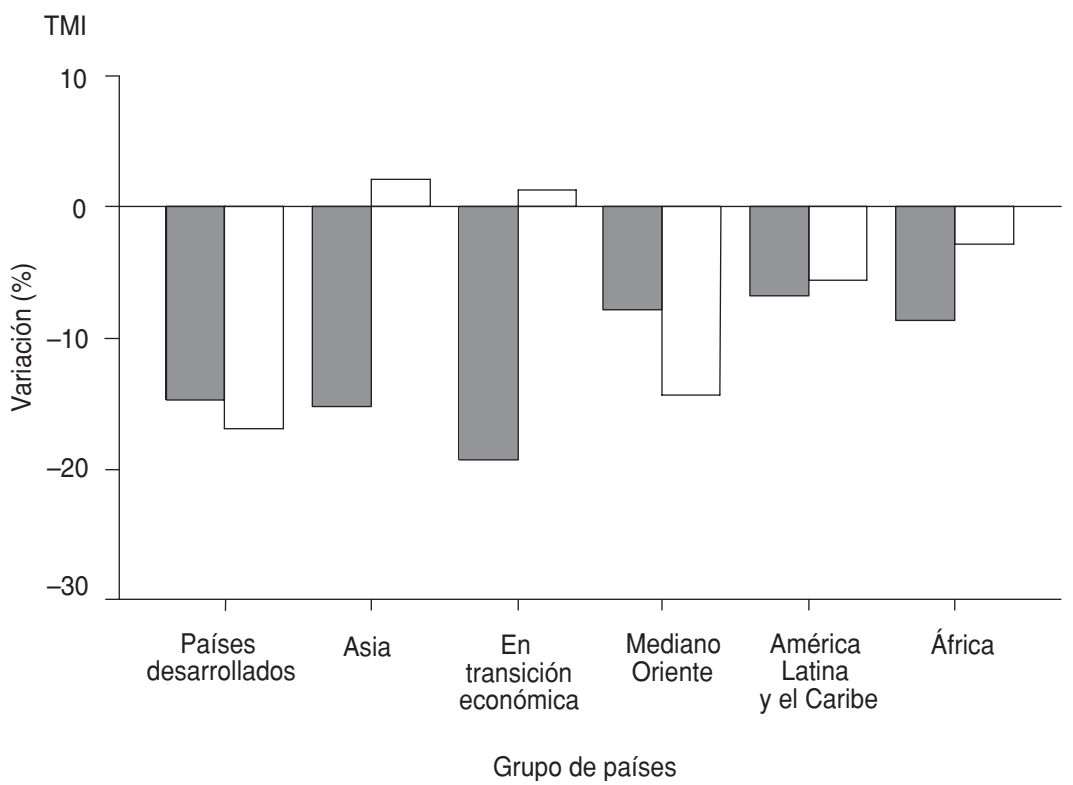

Fuente: Datos de la Organización Mundial de la Salud, las Naciones Unidas y el Programa de las Naciones Unidas para el Desarrollo (PNUD) (30-32).

En el modelo de regresión lineal multifactorial que contempla la EV y la MI, los estadísticos son significativos; ello se aplica tanto al modelo ge- neral $(F, P<0,001)$ como a los coeficientes $B(t, P<0,01)$, en lo referente a la variación en el gasto público entre 1980 y 1990. La asociación con el PIBpc no se mantiene e incluso se pierde la significación estadística en el modelo de la MI cuando aquel se incluye junto con el cambio en el gasto público durante el período de 1980-1990.

\section{DISCUSIÓN}

Los resultados de este estudio demostraron la ralentización de las tendencias favorables en dos importantes indicadores de salud a finales del siglo XX.

América Latina es el continente con la mayor ralentización en el incremento de la EV en comparación con otros grupos de países en desarrollo durante el período estudiado. Se observó el efecto acumulado de las fuertes medidas económicas aplicadas en los países latinoamericanos a finales del siglo pasado. Las variaciones en otros países de mayor desarrollo se podrían explicar por otras razones, como las migraciones de población que experimentaron a finales del siglo pasado y la dificultad cada vez mayor para seguir mejorando los indicadores de salud al mismo ritmo que antes. Estas interpretaciones coinciden con lo expuesto por otros autores que también constataron esta ralentización, si bien de manera menos clara y contundente. Weisbrot y colaboradores (11), por ejemplo, obtuvieron variaciones similares en todos los países y las atribuyeron a las transformaciones estructurales y a los cambios de políticas realizados durante el proceso de globalización, a partir de la década de 1970. Casas (34), en una investigación sobre América Latina, expone que a mayores ingresos se reduce la MI, pero también que una parte importante de la reducción de la mortalidad general no es atribuible a este factor sino otros elementos, como la información y las políticas sanitarias.

En el presente estudio, la MI en América Latina decreció menos que en los restantes grupos de países durante la década de 1980 (ralentización de la tendencia). Solo en la década siguiente, cuando el ajuste se extendió a otros países en desarrollo, los cambios en los indicadores de salud también se 
FIGURA 3. Diagrama de dispersión, indicadores de salud y tamaño del Estado. Países de América Latina y el Caribe, 2000

Esperanza de vida, en años

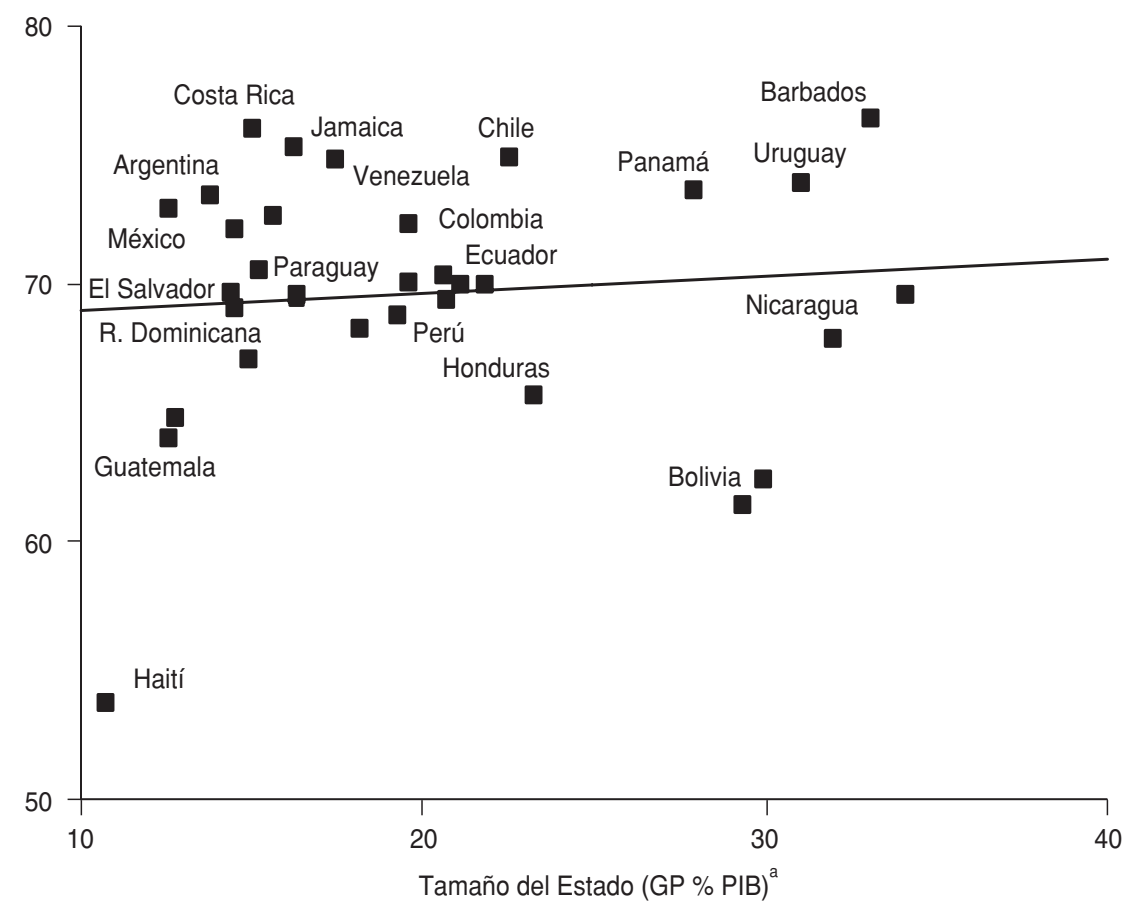

Tasa de mortalidad infantil (por 1000 nacidos vivos)

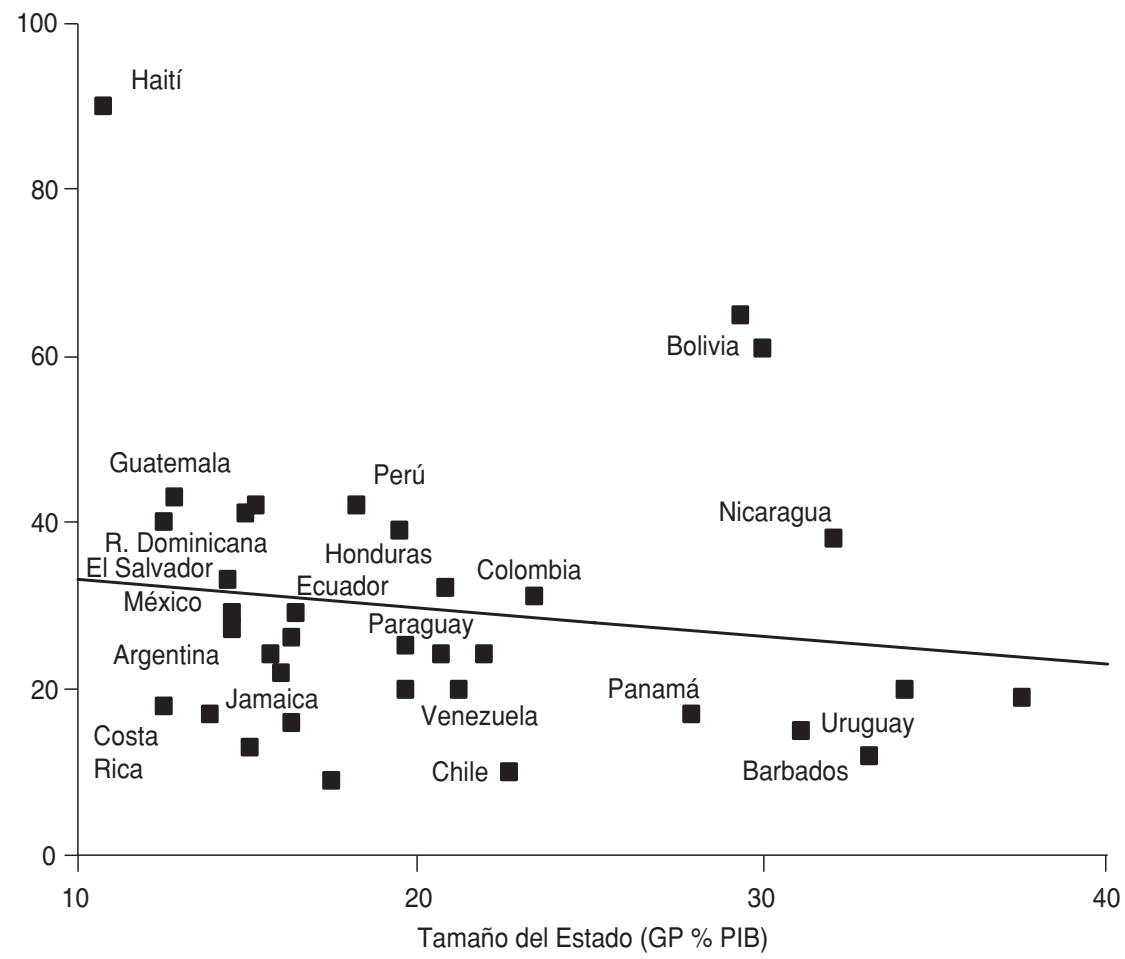

Fuente: Datos del Fondo Monetario Internacional, la Organización Mundial de la Salud y el Programa de las Naciones Unidas para el Desarrollo $(29,30,32)$

a Porcentaje del producto interno bruto (PIB) dedicado al gasto público (GP). vieron afectados negativamente en países de África, del sudeste asiático y del grupo en transición económica.

En particular, el gasto público (modelo de regresión lineal) se asocia de manera significativa con la MI. Resulta muy interesante esta relación entre los coeficientes de regresión del cambio en el gasto público durante el período de 1980-1990 con la MI después de ajustar según el PIBpc.

En América Latina fue donde primero se aplicaron las políticas neoliberales, cuyos principales motores han sido el ajuste estructural y el adelgazamiento del Estado. También fue ahí donde se sintió de manera más temprana el impacto macrosocial de esos ajustes sobre la salud, lo que demuestra que en solo una década de ajuste, las políticas macroeconómicas pueden causar efectos mensurables sobre los indicadores sociales.

No se puede olvidar, sin embargo, que ese efecto varía de acuerdo con la distribución de la riqueza en cada país, como señala Bartley (35). Es un hecho que la desigualdad en la distribución de los ingresos está asociada con la inequidad en el acceso a los servicios de salud y a otros servicios sociales importantes (36). Los niveles de salud de la población (EV, morbilidad y mortalidad) dependen directamente de las desigualdades en el ingreso y de otros factores sociales y culturalescorrelacionados (37).

Aunque el incremento de la EV se asocia con el incremento de la riqueza - reflejado en el PIBpc-, se insiste también en que hay un punto de inflexión en la tendencia del aumento de la EV en relación con el ingreso, es decir, un umbral a partir del cual es muy difícil seguir mejorando la EV aunque aumente el PIBpc. Sin embargo, este umbral no se ha alcanzado todavía en América Latina. Al contrario, los efectos de la reducción del tamaño del Estado en los países estudiados fueron más notorios en los países con mayores tasas de MI o una menor EV. Asimismo, los Estados más pequeños que tenían un menor gasto público y un menor PIBpc (las llamadas economías de bajos ingresos) presentaron una reducción porcentual más lenta de la MI con respecto a sus propias cifras de años anteriores. 
CUADRO 1. Correlación entre el adelgazamiento del Estado y los indicadores de salud en el mundo, 1980-2000

\begin{tabular}{lc}
\hline \multicolumn{1}{c}{ Indicador de salud } & Correlación de Pearson \\
\hline Esperanza de vida, quinquenio 1988-1992 & $0,191^{\mathrm{a}}$ \\
Tasa de mortalidad infantil, quinquenio 1988-1992 & $-0,234^{\mathrm{b}}$ \\
Esperanza de vida, quinquenio 1998-2002 & 0,140 \\
Tasa de mortalidad infantil, quinquenio 1998-2002 & $-0,211^{\mathrm{b}}$ \\
\hline
\end{tabular}

Fuente: Datos del Fondo Monetario Internacional y la Organización Mundial de la Salud (29, 30).

a Nivel de significación: 0,05 (bilateral).

${ }^{\mathrm{b}}$ Nivel de significación: 0,01 (bilateral).

Este estudio presenta algunas limitaciones. Por ser de tipo ecológico, la unidad de observación y análisis está constituida por los Estados y aunque permite detectar asociaciones a partir de datos agregados sobre factores políticos y económicos, no permite hacer predicciones, generalizaciones o suposiciones acerca de países individuales. Además, por basarse en datos agregados, las asociaciones con el PIBpc de los países son mayores y más intensas, lo cual impide llegar a conclusiones más claras. En los estudios de corte transversal, las correlaciones de los indicadores de salud con macroindicadores económicos no siempre son precisas (38), por lo que se recomienda hacer un análisis de tendencias que suministre información adicional, como el efecto histórico de las variables sociopolíticas sobre la salud.

A pesar de que los resultados presentados evaluaron la exposición de los servicios de salud a las políticas macroeconómicas solamente durante 20 años (1980-2000), se pudieron hacer algunas comparaciones con datos anteriores de las variables explicativas. Aunque los efectos no se pudieron contrastar suficientemente, se logró demostrar una relación de efecto retardado de las variables independientes (factores económicos) sobre las dependientes (indicadores de salud). No fue posible hacer aseveraciones de causalidad ni descartar que el efecto pueda atribuirse a otras causas, como los rezagos de la decadencia del modelo anterior o el efecto deletéreo inmediato de una política muy severa, que a más largo plazo podría llegar a expresar ciertas bondades (de acuerdo con los defensores del ajuste neoliberal). Sin embargo, hasta el momento esos efectos benéficos no se pueden medir ni predecir.

El mejoramiento de la salud es mayor en los países desarrollados, que ostentan mejores niveles de protección social y económica para su población, mientras que en los países pobres es mucho más moderado y se sienten más fuerte y rápidamente las medidas y políticas macroeconómicas implementadas. Otros autores han señalado este mismo resultado en estudios de grupos poblacionales (39).

En conclusión, en la década de 1980 el ajuste de las políticas macroeconómicas tuvo un efecto negativo sobre los indicadores sociales, específicamente sobre los relacionados con las condiciones de salud en América Latina y el Caribe. Estos efectos persistieron durante la década siguiente y en casi todas las regiones del mundo. El adelgazamiento del Estado tuvo un efecto negativo sobre la MI y la EV en América Latina y el Caribe

En consecuencia, se recomienda redefinir las políticas que guiaron el ajuste estructural al final del siglo pasado y que algunos gobiernos aún continúan aplicando. Se deben diseñar políticas que favorezcan el desarrollo social de las comunidades y de la población de nuestros países - sin desconocer el desarrollo institucional- y reorientar el gasto público poniendo énfasis en una política social de desarrollo.

CUADRO 2. Regresión lineal: Adelgazamiento del Estado (cambio en gasto público) e indicadores de salud en América Latina, década de 1980-1990

\begin{tabular}{lcccc}
\hline \multicolumn{1}{c}{ Indicador de salud } & $\begin{array}{c}\text { Coeficiente } \\
\text { de Pearson }\end{array}$ & $\begin{array}{c}\text { Coeficiente de } \\
\text { determinación }\left(R^{2}\right)\end{array}$ & $\begin{array}{c}\text { Coeficiente de } \\
\text { regresión }(B)\end{array}$ & $\begin{array}{c}\text { Significación } \\
\text { estadística }(P)\end{array}$ \\
\hline $\begin{array}{l}\text { Tasa de mortalidad infantil } \\
\text { por 1 000 nacidos } \\
\text { vivos, quinquenio 1988-1992 }\end{array}$ & 0,556 & 0,336 & $-0,554$ \\
$\begin{array}{l}\text { Esperanza de vida, quinquenio } \\
1988-1992 \text { (años) }\end{array}$ & 0,641 & 0,410 & 0,072 & 0,008 \\
\hline
\end{tabular}

Fuente: Datos del Fondo Monetario Internacional y de la Organización Mundial de la Salud (29, 30)

a Modelo de regresión lineal multifactorial, ajustado según el producto interno bruto per cápita. 


\section{REFERENCIAS}

1. Centro Latinoamericano de Administración para el Desarrollo. Una nueva gestión pública para América Latina. Documento preparado por el Consejo Científico del CLAD [versión electrónica]; 1998. Hallado en: http:// unpan1. un.org/intradoc/groups/public/documents/ CLAD/UNPAN000161.pdf. Acceso el 2 de mayo 2004.

2. Hayek F. Los fundamentos de la libertad. Madrid: Unión Editorial; 1998.

3. Sotelo A. Globalización: ¿estancamiento o crisis en América Latina? Rev Problemas del Desarrollo (IIEc-UNAM), México. 2000;120: 31-53. Hallado en: http://alainet.org/active/ 1666. Acceso el 12 de mayo de 2006

4. Banco Interamericano de Desarrollo. América Latina frente a la desigualdad. Informe 19981999. Washington, D.C.: BID; 1998.

5. Alianza Social Continental. Declaración de la ASC sobre el ALCA. América Latina en Movimiento 2001 [versión electrónica]; 2002. Hallado en: http://alainet.org/active/1626. Acceso el 15 de mayo de 2006.

6. Williamson J. What Washington means by policy reform. En: J Williamson, ed. Latin American adjustment: how much has happened? Washington, D.C.: Institute for International Economics; 1990. Pp. 7-38.

7. Castles F. The future of the welfare state: crisis myths and crisis realities. Int J Health Serv. 2002;32(2):255-77.

8. Mesa-Lago C. Desarrollo social, reforma del Estado y de la seguridad social al umbral del siglo XXI. Documento del CLAD [versión electrónica]; 2000. Hallado en: http://www. clad.org.ve/mesalago.html. Acceso el 23 de abril de 2006.

9. Banco Mundial. Hacia un Estado más eficiente y de menor dimensión. En: Banco Mundial. Informe sobre el desarrollo mundial, 1996. De la planificación centralizada a la economía de mercado. Washington, D.C.: BM; 1996. Pp. 133-48.

10. Banco Interamericano de Desarrollo. Proceso económico y social en América Latina. Informe 1996. ¿Cómo organizar con éxito los servicios sociales? Washington, D.C.: BID; 1996.

11. Weisbrot M, Baker D, Kraev E, Chen J. The scorecard on globalization 1980-2000: its consequences for economic and social well-being. Int J Health Serv. 2002;32(2):229-53.

12. Bolis M, Arboleda J, Stuart HL, Freeman P González MA. Acceso a los servicios de salud en el marco del TLC. Washington, D.C.: Organización Panamericana de la Salud; 1999.
13. Kickbusch I, de Leeuw E. Global public health: revisiting healthy public policy at the global level. Health Promot Int. 1999;14:285-8.

14. Franco A. Globalizar la salud. Gaceta Sanitaria (Barcelona); 2003;17(2):157-63.

15. Kunitz SJ. Globalization, states, and the health of indigenous peoples. Am J Public Health. 2000;90:1531-9.

16. Navarro V. Health and equity in the world in the era of "globalisation". Int J Health Serv. 1999;29:215-26.

17. Acheson D. Independent inquiry into inequalities in health: report. London: HMSO; 1998.

18. Black D, Morris JN, Townsend P. Inequalities in health: The Black Report and the health divide. Harmonsworth (U.K.): Penguin; 1982.

19. Judge K. Income distribution and life expectancy: a critical appraisal. Br Med J. 1995; 311:1282-5.

20. Sen A. Inequality explained. Cambridge (Massachusetts): Harvard University Press; 1992.

21. Sen S. Mortality and distribution and income. Br Med J. 1998;316:1611-2.

22. Wilkinson RG. National mortality rates: the impact of inequality. Am J Public Health. 1992;82:1082-4

23. Musgrove P, Zeramdini R, Carrin G. Basic patterns in national health expenditure. Bull WHO. 2002;80:134-42.

24. Musgrove P. La eficacia en función de los costos y la reforma del sector salud. Salud Publica Mex. 1995;37:363-74.

25. Filmer D, Pritchett L. The impact of public spending on health: does money matter? Soc Sci Med. 1999;49:1309-23.

26. United Nations, Economic and Social Council. Basic data on government expenditure and taxation. Report of the Secretariat, Committee of Experts on Public Administration, Third session. New York: UN; 2004. Hallado en: http://unpan1.un.org/intradoc/groups/pub lic/documents/UN/UNPAN015272.pdf. Acceso el 13 de mayo de 2006.

27. Banco Mundial. Informe sobre el desarrollo mundial, 1997. El Estado en un mundo en transformación. Washington, D.C.: Oxford University Press; 1997.

28. Franco A, Gil D, Álvarez-Dardet C. Tamaño del Estado (gasto público) y salud en el mundo, 1990-2000. Gaceta Sanitaria (Barcelona). 2005;19(3):186-92.

29. International Monetary Fund. Government finances statistics yearbook 2000. Washington, D.C.: IMF; 2000
30. World Health Organization. The World Health Report. Health systems: improving performance. Geneva: WHO; 2001. Hallado en: www.who.int/whr2001/2001/archives/ 2000/en/press release.htm. Acceso el 13 de mayo de 2006

31. United Nations. Demographic yearbook 1999. Issue 51. New York: UN; 2000.

32. Programa de Naciones Unidas para el Desarrollo. Informe de desarrollo humano 2002 Profundizar la democracia en un mundo fragmentado. New York: PNUD/Ediciones Mundiprensa; 2002.

33. Franco A. Contexto político, Estado y salud en el mundo. [Tesis doctoral]. Alicante (España): Universidad de Alicante; 2004.

34. Casas-Zamora JA. Salud, desarrollo humano y gobernabilidad en América Latina y el Caribe a inicios del siglo XXI. Rev Panam Salud Publica. 2002;11(5-6):397-408.

35. Bartley M. Health inequality. An introduction to theories, concepts and methods. Cambridge (U.K.): Polity Press; 2004.

36. Dach N. Inequidades en salud: cómo estudiarlas. En: Restrepo H, Málaga H. Promoción de la salud: cómo construir una vida saludable. Santa Fe de Bogotá: Editorial Médica Panamericana; 2001. Pp. 84-104.

37. Deaton A. Health, inequality, and economic development. Geneva: Commission on Macroeconomics and Health; 2001. (CMH Working Paper Series No. WG1: 3). Hallado en: http://www.cmhealth.org/docs/wg1_ paper3.pdf. Acceso el 23 de abril de 2006

38. Van PJ. Applications of summary measures of population health. En: Murray CJ, Salomon JA, Colin D, Mathers CD, López A, eds. Summary measures of population health: concepts, ethics, measurement and applications. Geneva: World Health Organization; 2002. Pp. 53-60.

39. Vega J, Hollstein RD, Delgado I, Marshall G, Yach D. Social inequalities and health in an intermediate-development nation: education and adult mortality in Chile, 1980-1996. En: Evans T, Whitehead M, Diderichsen F, Bhuiya A, Wirth $\mathrm{M}$, eds. Challenging inequities in health: from ethics to action. New York: Oxford University Press; 2001. Pp. 123-37.

Manuscrito recibido el 11 de agosto de 2005. Aceptado para publicación, tras revisión, el 11 de abril de 2006. 
ABSTRACT Objective. To assess the impact of structural adjustment on health indicators in Latin America and the Caribbean during 1980-2000.

The effect of structural adjustment on health conditions in Latin America and the Caribbean, 1980-2000

Methods. This was an ecological study. Public spending and per capita gross domestic product (pcGDP) figures were obtained from the World Bank, and life expectancy (LE) and infant mortality (IM) figures were obtained from the World Health Organization. Structural adjustment (government downsizing) was assessed by looking at the change in the amount of spending taken up by the government (or the reduction in public spending) in Latin American and Caribbean countries during 1980-1990. Changes in health indicators were measured in terms of the percentage variation in LE and IM. The variations found in Latin America and the Caribbean were compared to those seen in different groups of countries in other parts of the world during 1980-2000. Pearson's chi squared test was used to explore the associations between the decrease in public spending and health indicators. In order to estimate the health effects of such changes, a multivariate linear regression model was created, with adjustments for pcGDP.

Results. A deceleration in the rise of LE and in the decline of IM in Latin America and the Caribbean was noted, especially over the period from 1980 through 1990. Significant associations were observed between health indicators and the change in public spending in all groups of countries included in the study. When adjustments were introduced into the multiple regression model, the only associations that remained were seen in Latin America and the Caribbean.

Conclusions. In the decade of 1980, adjustments in macroeconomic policies had a negative effect on social indicators, specifically those that had to do with health conditions in Latin America and the Caribbean. Such an effect lasted throughout the following decade.

Key words State reform, reduction in public spending, impact in the health condition, Latin America, Caribbean region. 